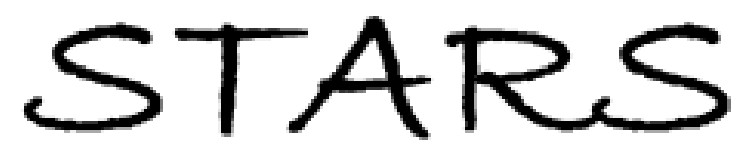

University of Central Florida

STARS

$1-1-1992$

\title{
Pressure-Dependence Of The Hyperfine Field Of A Cd Probe In A Relaxed Trivacancy Complex In Ni
}

Lee Chow

University of Central Florida

Xin Zhao

University of Central Florida

Gary S. Collins

Find similar works at: https://stars.library.ucf.edu/facultybib1990

University of Central Florida Libraries http://library.ucf.edu

This Article is brought to you for free and open access by the Faculty Bibliography at STARS. It has been accepted for inclusion in Faculty Bibliography 1990s by an authorized administrator of STARS. For more information, please contact STARS@ucf.edu.

\section{Recommended Citation}

Chow, Lee; Zhao, Xin; and Collins, Gary S., "Pressure-Dependence Of The Hyperfine Field Of A Cd Probe In A Relaxed Trivacancy Complex In Ni" (1992). Faculty Bibliography 1990s. 421.

https://stars.library.ucf.edu/facultybib1990/421

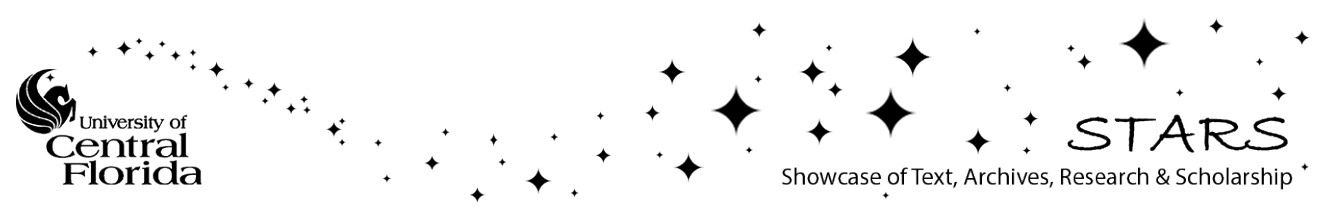




\title{
Pressure dependence of the hyperfine field of a $\mathrm{Cd}$ probe in a relaxed trivacancy complex in $\mathrm{Ni}$
}

\author{
Lee Chow and Xin Zhao \\ Department of Physics, University of Central Florida, Orlando, Florida 32816 \\ Gary S. Collins \\ Department of Physics, Washington State University, Pullman, Washington 99164-2814
}

(Received 29 August 1991)

\begin{abstract}
Perturbed $\gamma-\gamma$ angular-correlation experiments were carried out using extremely dilute ${ }^{111} \mathrm{Cd}$ probes to measure the pressure dependence of the magnetic hyperfine field at a vacancy-solute complex in $\mathrm{Ni}$ in which the Cd probe is relaxed to the center of a tetrahedron of vacancies. The pressure dependence of the hyperfine field of the defect-free substitutional site was found to be $d H_{\mathrm{hf}} / d p=-0.038(6) \mathrm{kG} / \mathrm{kbar}$, which agrees with a previous measurement. The pressure dependence of the hyperfine field associated with the vacancy complex was found to be $d H_{\mathrm{hf}} / d p=-0.023(4) \mathrm{kG} / \mathrm{kbar}$. Results are not found to be consistent with a simple picture of the hyperfine-field-production mechanism obtained from recent firstprinciples calculations.
\end{abstract}

\section{INTRODUCTION}

Magnetic hyperfine fields at nuclei of diamagnetic impurities in ferromagnetic hosts have been well studied for the past thirty years. It has been noticed from the analysis of tabulated fields ${ }^{1-3}$ that there are general trends in impurity hyperfine fields across the periodic table; in particular, for series of $s p$ impurities such as $\mathrm{Ag}$ to $\mathrm{Xe}$, the fields are negative at the beginning of the series, change over to positive near the middle, and reach a steep maximum near the end. Several models have been proposed to explain this trend in terms of the Ruderman-Kittel-Kasuya-Yosida- (RKKY) like model, ${ }^{4}$ volume-misfit model, ${ }^{5}$ and lattice-distortion model. ${ }^{6}$ It has also been emphasized ${ }^{7}$ that detailed pressure and temperature dependence measurements of the $s p$ impurity hyperfine fields should elucidate the mechanisms behind the systematic trends.

In recent years, studies have also been made of impurity hyperfine fields when the impurity probe traps vacancies in its first-neighbor shell. Hohenemser et al. ${ }^{8} \mathrm{ob}-$ served a cubic-symmetric defect-associated $\mathrm{Cd}$ hyperfine field of $-27 \mathrm{kG}$ by perturbed $\gamma-\gamma$ angular correlation (PAC) in ${ }^{111}$ In-implanted $\mathrm{Ni}$, which is more positive than the $-67-\mathrm{kG}$ hyperfine field at substitutional defect-free Cd. They identified the Cd-vacancy complex as a solute associated with three vacancies, in which the position of the substitutional solute relaxed to a tetrahedral interstitial site in fcc $\mathrm{Ni}$ surrounded by four vacancies. This is denoted as the tetrahedral complex below. Because of the cubic symmetry of the probe in this complex, no quadrupole interactions are present, and there is only a magnetic interaction. What makes the tetrahedral complex interesting is the difference between the numbers and distances of the $\mathrm{Ni}$ magnetic moments from the $\mathrm{Cd}$ probe in the substitutional and tetrahedral sites. Comparison of their magnetic fields and their pressure dependence may give insight into the origin of the transferred hyperfine field.
Later on, de Waard et al. ${ }^{9}$ used ${ }^{119} \mathrm{Sb}$ implantation to study fields at vacancy-Sn complex in $\mathrm{Ni}, \mathrm{fcc} \mathrm{Co}$, and $\mathrm{Fe}$. In all three cases, they found that the vacancy-associated complexes have increased (more positive) ${ }^{119} \mathrm{Sn}$ impurity hyperfine fields. They interpreted their data using the conduction-electron-polarization (CEP) model of Blandin and Campbell ${ }^{4}$ and obtained good qualitative agreement. More recently, Weyer and collaborators ${ }^{7,11,12}$ and de Waard and collaborators ${ }^{9,10}$ both carried out detailed studies of the tetrahedral complexes involving ${ }^{119} \mathrm{Sn}$, ${ }^{119} \mathrm{Sb},{ }^{121} \mathrm{Te},{ }^{129} \mathrm{Te}$ Mössbauer probes, ${ }^{7,10,11}$ and a ${ }^{111} \mathrm{Cd}$ PAC probe. ${ }^{12}$

In this paper, we use perturbed angular correlations of ${ }^{111} \mathrm{Cd}$ to study the pressure dependence of the $\mathrm{Cd}$ hyperfine field at the tetrahedral complex in $\mathrm{Ni}$. We compare it to the pressure dependence at the defect-free substitutional site and discuss results in terms of recent calculations by Akai, Akai, and Kanomori. ${ }^{13}$

\section{EXPERIMENT AND RESULTS}

Samples used were $25-\mu \mathrm{m}$-thick, $99.999 \%$ pure Ni foils from Johnson Matthey Co. which were cleaned in diluted $\mathrm{HCl}$ solution and rinsed with deionized water. Carrierfree ${ }^{111}$ In activity in $0.05 \mathrm{M} \mathrm{HCl}$ solution was purchased from New England Nuclear Corporation and deposited and dried on the $\mathrm{Ni}$ foils. The foils were then annealed under flowing $\mathrm{H}_{2}$ at $800^{\circ} \mathrm{C}$ for $1 \mathrm{~h}$ and oven cooled to diffuse the activity uniformly inside. A preliminary PAC measurement was made to ensure that most of the ${ }^{111}$ In activity stays at the substitutional site. The foil was then cold-rolled at room temperature to about twice its original length and annealed for $15 \mathrm{~min}$ at $500 \mathrm{~K}$ in vacuum to form the tetrahedral complex by migration and trapping of trivacancies. ${ }^{14}$ A PAC measurement of the damaged foil was taken at ambient pressure and served as a zero-pressure standard. Different samples were used for each pressure measurement. Typical values of the site fractions for the substitutional and tetrahedral complexes 

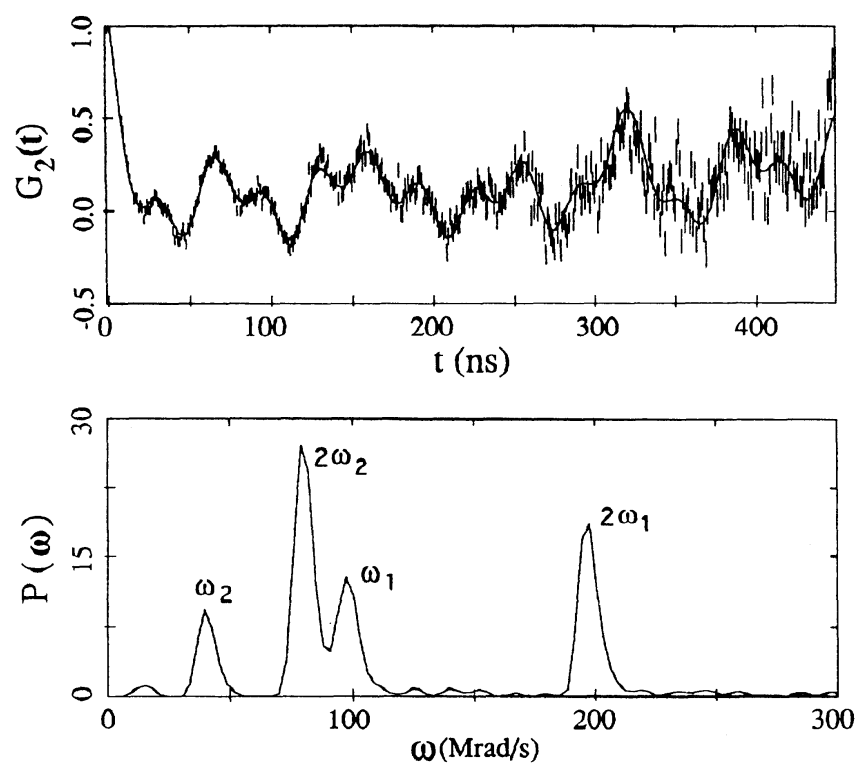

FIG. 1. Typical zero-field PAC spectrum and its Fourier spectrum of ${ }^{111} \mathrm{Cd}$ in $\mathrm{Ni}$ after deformation and annealing at 500 $K$ showing the $\omega$ and $2 \omega$ signals of the substitutional and tetrahedral complex sites.

in the damaged foils were $25 \%$ and $20 \%$, respectively. Afterward, high-pressure measurements $(P>20 \mathrm{kbar})$ were carried out using an opposed Bridgman anvil cell ${ }^{15}$ capable of presures of $70 \mathrm{kbar}$. The pressure in the Bridgman anvil cell was loaded through a 25 -ton press. The cell was supported at an upper shoulder; this allowed the cell to stretch downward upon loading. A dial indicator was used to measure the relative stretch of the cell. The pressure was locked onto the sample by tightening a nut on the top of the pressure cell. The hydraulic pressure of the press was then released slowly. The nut retained most of the pressure on the sample. Usually the pressure dropped approximately $10 \%$ after the nut was tightened and the hydraulic pressure relased. The average pressure in the cell was calculated by the ratio of the applied force to the anvil area. The spot size of the ${ }^{111}$ In activity was typically $2 \mathrm{~mm}$ in diameter and was centered

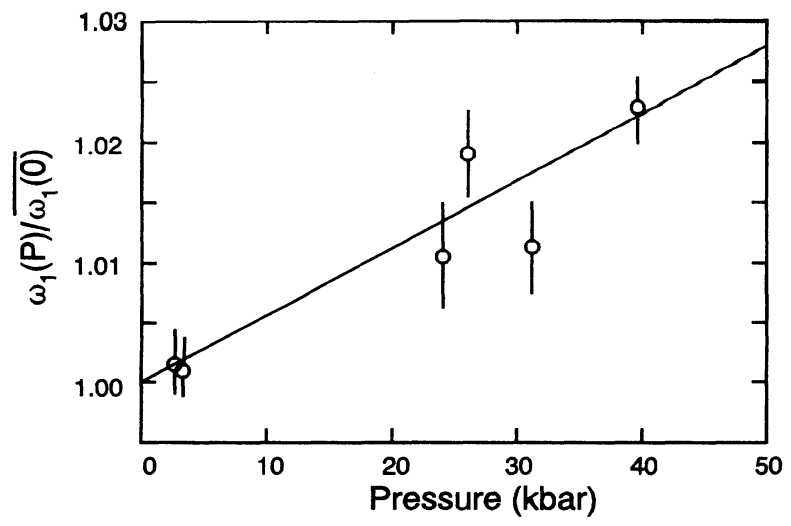

FIG. 2. Pressure dependence of the hyperfine field of substitutional $\mathrm{Cd}$ in $\mathrm{Ni}$.

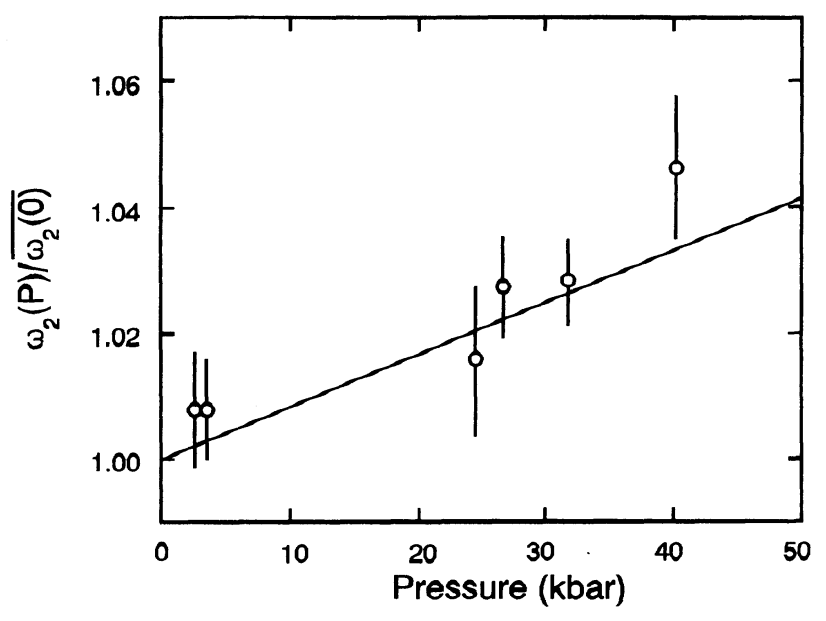

FIG. 3. Pressure dependence of the $\mathrm{Cd}$ hyperfine field in the tetrahedral complex in $\mathrm{Ni}$.

in the 10-mm-diam anvil face. The pressure measurements at lower pressures $(P<7 \mathrm{kbar})$ were carried out using a hydrostatic pressure pump equipped with an intensifier. The pressures were measured by an Ashcroft pressure gauge. PAC measurements were made using two $\mathrm{NaI}$ detectors at an angle of $180^{\circ}$. Coincidence spectra were first fitted with a nonlinear least-squares fitting program to determine the accidental background. Then the raw data were background subtracted and divided by the nuclear decay lifetime to obtain the perturbation function. Standard methods ${ }^{16}$ were used to extract the nuclear Larmor precession frequency $\omega$ and the site population from the reduced data. Hyperfine fields were calculated from the Larmor frequencies using the known $g$ factor ${ }^{17}$ of the PAC level of ${ }^{111} \mathrm{Cd}$.

All measurements were made in zero applied field. In Fig. 1, a typical zero-pressure PAC time-domain spectrum is shown with its Fourier transform. Since the data were taken in zero applied field, signals at both the Larmor frequency $\omega$ and $2 \omega$ are present for each site. The pressure dependences of the fields at the substitutional site (site 1) and the tetrahedral complex (site 2) are shown in Figs. 2 and 3. The measured Larmor frequencies were normalized by the average Larmor frequencies measured at zero pressure. In Fig. 2, we find the pressure dependence to be $-0.038(6) \mathrm{kG} / \mathrm{kbar}$ from the slope of a straight-line fit, in agreement with a previous measurement of Lindgren and Vijay. ${ }^{18}$ For the tetrahedral complex, the corresponding result in Fig. 3 is -0.023(4) $\mathrm{kG} / \mathrm{kbar}$. Results of our experiment are summarized in Table I.

\section{DISCUSSION}

Because of their complexity, there have been very few first-principles calculations ${ }^{13,19}$ of impurity hyperfine field in ferromagnetic hosts. Lindgren and Ellis ${ }^{19}$ used the local-density formalism in an embedded-cluster model to calculate impurity hyperfine fields in $\mathrm{Fe}, \mathrm{Co}$, and $\mathrm{Ni}$. Akai, Akai, and Kanomori ${ }^{13}$ used Green-function methods to calculate $s p$ and transition-metal impurity 
TABLE I. Substitutional and tetrahedral complex-associated hyperfine fields and pressure derivatives of Cd in Ni measured at 293 K.

\begin{tabular}{llccc}
\hline & $H_{\mathrm{hf}}(P=0)$ & $d H_{\mathrm{hf}} / d P$ & $d \ln H_{\mathrm{hf}} / d P$ & $d \ln H_{\mathrm{hf}} / d \ln V^{a}$ \\
\hline $\begin{array}{l}\text { Substitutional } \\
\text { Tetrahedral }\end{array}$ & $-66.9(3) \mathrm{kG}$ & $-0.038(6) \mathrm{kG} / \mathrm{kbar}$ & $+0.56(8) \times 10^{-3} \mathrm{kbar}^{-1}$ & $-1.04(15)$ \\
complex & $-27.3(3) \mathrm{kG}$ & $-0.023(4) \mathrm{kG} / \mathrm{kbar}$ & $+0.83(15) \times 10^{-3} \mathrm{kbar}^{-1}$ & $-1.54(28)$ \\
\hline
\end{tabular}

${ }^{\mathrm{a}}$ Calculated using the bulk compressibility of $\mathrm{Ni} c=-5.4 \times 10^{-4} \mathrm{kbar}^{-1}$ is from Ref. 23 .

hyperfine fields in ferromagnetic hosts. Both calculations gave similar results which explain the hyperfine-field systematics for substitutional impurities. These calculations have led to the following qualitative picture. Generally speaking, the electronic spin polarization at the nucleus of an $s p$ impurity, which in turn leads to the hyperfine field through the Fermi contact term, is induced through hybridization between impurity $s$ and host $d$ states. Because of this hybridization, the local spin density of states at the nucleus splits into bonding and antibonding states. Because of the exchange interaction, the spin polarization at the nucleus produced by the bonding states is negative and that of the antibonding states is positive. Thus, the net hyperfine field is a competition between two large contributions with opposite sign.

When pressure is applied specifically to $\mathrm{Ni}$, there are two major mechanisms that change the hyperfine field: (1) The magnetic moment of the Ni host is observed by Kondorskii and $\operatorname{Sedov}^{20}$ to decrease, which implies a decrease in the magnitude of the hyperfine field, no matter what its sign. (2) The matrix element mixing the host $d$ states and the impurity $s$ states increases due to increasing overlap of orbitals, pushing some antibonding states above the Fermi level. This leads a more negative magnetic hyperfine field.

For a positive total hyperfine field, the two mechanisms both tend to decrease the field, while for a negative total field, the mechanisms oppose each other. This explains why in the cases ${ }^{21,22}$ of ${ }^{125} \mathrm{TeFe}$ and ${ }^{129} \mathrm{IFe}$, where the fields are positive, the pressure derivatives of the hyperfine field ${ }^{18}$ are much larger than for ${ }^{111} \mathrm{CdFe}$ or ${ }^{111} \mathrm{CdNi}$.

In the present work, the pressure derivatives of the
${ }^{111} \mathrm{CdNi}$ hyperfine fields at the substitutional and tetrahedral vacancy sites are both negative, which indicates the second mechanism is more important than the first.

Intuitively, we expect the local compressibility at the vacancy complex to be larger than the bulk compressibility of nickel. As the pressure increases, therefore, the $\mathrm{Ni}$ host atom distances from the solute should decrease more rapidly for the vacancy complex than for the defect-free site. Relatively, this will enhance the second mechanism for the vacancy complex, so that we would expect a larger pressure derivative for the vacancy complex. However, our results clearly showed that the pressure derivative at the vacancy complex was only half of that at the substitutional site. Thus, other factors not considered in the simple picture above must affect the pressure derivative of the hyperfine field, such as the details structure of the density of states near the Fermi surface. To understand our results, first-principles calculations of hyperfine fields and pressure derivatives at both substitutional and vacancy complex sites appear to be necessary. For the vacancy-associated sites, these should include effects of local lattice relaxation at both sites.

\section{ACKNOWLEDGMENTS}

We thank T. Kimble of University of Central Florida, and S. L. Shropshire of Washington State University for technical help at early stages of this work. The work done at Washington State University was supported in part by the National Science Foundation under Grant No. DMR 86-19688.
${ }^{1}$ A. Shirley and G. A. Westenberger, Phys. Rev. 138, A170 (1965).

${ }^{2}$ G. N. Rao and A. K. Singhvi, Phys. Status Solidi B 84, 9 (1977).

${ }^{3}$ G. N. Rao, Hyperfine Interact. 24-26, 1119 (1985).

${ }^{4}$ E. Daniel and J. Friedel, J. Phys. Chem. Solids 24, 1601 (1963); A. Blandin and I. A. Campbell, Phys. Rev. Lett. 31, 51 (1973).

${ }^{5}$ M. B. Sterns, Phys. Rev. B 13, 4180 (1976); 13, 1183 (1976); 8, 4383 (1973).

${ }^{6}$ B. Kolk, Hyperfine Interact. 4, 313 (1978).

${ }^{7}$ G. Weyer, Hyperfine Interact. 51, 901 (1989).

${ }^{8}$ C. Hohenemser, A. R. Arends, H. de Waard, H. G. Devare, F. Pleiter, and S. A. Drentje, Hyperfine Interact. 3, 297 (1977).

${ }^{9}$ H. de Waard, D. W. Hafemeister, L. Niesen, and F. Pleiter, Phys. Rev. B 24, 1274 (1981).
${ }^{10}$ H. de Waard, G. L. Zhang, and R. H. Huizenga, Hyperfine Interact. 52, 229 (1989).

${ }^{11}$ G. Weyer, F. T. Pedersen, and H. Grann, Nucl. Instrum. Methods Phys. Res. B 7/8, 103 (1985).

${ }^{12}$ F. Raether, G. Weyer, K. P. Lieb, and J. Chevallier, Phys. Lett. A 131, 471 (1988).

${ }^{13}$ M. Akai, H. Akai, and Kanomori, J. Phys. Soc. Jpn. 54, 4246 (1985).

${ }^{14}$ Gary S. Collins and Reinhardt B. Schuhmann, Hyperfine Interact. 15/16, 391 (1983).

${ }^{15}$ W. T. Krakow, W. D. Josephson, P. A. Deane, D. L. Willianson, and L. D. Roberts, Phys. Rev. B 23, 499 (1981).

${ }^{16}$ A. R. Arend, C. Hohenemser, F. Pleiter, H. de Waard, L. Chow, and R. M. Suter, Hyperfine Interact. 8, 191 (1980).

${ }^{17}$ H. Bertschat, H. Haas, F. Pleiter, E. Recknagel, E. 
Schlodder, and B. Spellmeyer, Z. Phys. 270, 203 (1974).

${ }^{18}$ B. Lindgren and Y. K. Vijay, Hyperfine Interact. 9, 379 (1981).

${ }^{19}$ B. Lindgren and D. E. Ellis, Phys. Rev. B 26, 636 (1982).

${ }^{20}$ E. I. Kondorskii and V. I. Sedov, Zh. Eksp. Teor. Fiz. 38, 773 (1960) [Sov. Phys. JETP 11, 561 (1960)].
${ }^{21}$ U. Ladwig, B. Perscheid, and G. Kaindl, Hyperfine Interact. 9, 425 (1981).

${ }^{22}$ K. Frank, U. Ladewig, B. Perscheid, G. Kaindl, S. Reintsema, and H. Pattyn, J. Magn. Magn. Mater. 15-18, 641 (1980).

${ }^{23}$ G. A. Alers, J. R. Neighbours, and H. Sato, J. Phys. Chem. Solids 31, 986 (1960). 